\title{
Phenotypic and genetic changes in the life cycle of small colony variants of Salmonella enterica serotype Typhimurium induced by streptomycin
}

Wanli Li ${ }^{1}$, Yinghui Li ${ }^{2}$, Yarong Wu ${ }^{3}$, Yujun Cui ${ }^{3}$, Yao Liu ${ }^{1}$, Xiaolu Shi ${ }^{2}$, Qian Zhang ${ }^{2}$, Qiongcheng Chen ${ }^{2}$, Qun Sun ${ }^{\text {* }}$ and Qinghua $\mathrm{Hu}^{2 *}$

\begin{abstract}
Background: Small colony variants (SCVs), constituting a slow-growing subpopulation of bacteria that facilitates persistence in lethal environmental conditions, are able to revert to the phenotype of rapid growth for further proliferation and transmission. Salmonella enterica serotype Typhimurium is one of the most important foodborne pathogens. This study investigated the genetic mechanisms how SCVs induced by streptomycin reverted to the fast-growing phenotype and the phenotypic changes of SCVs among their complete life cycle in S. Typhimurium.
\end{abstract}

Methods: Salmonella Typhimurium SCVs were obtained by streptomycin treatment and their revertants were collected in the absence of antibiotics. The fitness, antimicrobial susceptibility, biofilm formation, and the biofilm-related genes expression were analyzed in comparison to their wild type strain, and the whole genome sequencing was performed to identify the genetic changes in the life cycle of S. Typhimurium SCVs.

Results: Small colony variants were characterized by an increased antimicrobial resistance to streptomycin (64-fold), imipenem (twofold), and gentamicin (fourfold). A significant increase in biofilm production with higher expression of csgB was observed in SCVs $(P<0.01)$. The genetic alterations of all SCVs occurred in ubiE gene (coenzyme $\mathrm{Q}_{8}$ and menaquinone synthesis) with frameshift mutations. However, all fast-growing revertants again lost the trait of increased biofilm production $(P>0.05)$, in which two modes of the genetic changes for reversing to the rapidly growing form were observed: four revertants harbored a secondary mutation in $u b i E$, which reinstated most of the amino acid sequence of the $u b i E$, and other four revertants harbored a mutation in prfB.

Conclusions: Salmonella Typhimurium could switch to the phenotype of SCVs under the treatment of streptomycin by a mutation in ubiE, partially combined with increased production of biofilm, and these SCVs could escape from growth restriction by a compensatory mutation in prfB or a new mutation in ubiE. These findings may contribute to establishing phenotype-directed treatments against SCVs of S. Typhimurium.

Keywords: Small colony variants, Salmonella, Biofilm, ubiE, prfB

\footnotetext{
*Correspondence: qunsun@scu.edu.cn; huqinghua03@163.com

${ }^{1}$ Key Laboratory of Bio-resources and Eco-environment of the Ministry

of Education, College of Life Sciences, Sichuan University, 29\# Wangjiang

Rd., Chengdu, Sichuan 610064, P. R. China

2 Shenzhen Major Infectious Disease Control Key Laboratory, Shenzhen

Centre for Disease Control and Prevention, Shenzhen, Guangdong

518055, P. R. China

Full list of author information is available at the end of the article
} 


\section{Background}

Small colony variants (SCVs), an aberrant form of bacterial phonotype, constitute a slow-growing subpopulation typically characterized with reduced metabolic activity and increased resistance to antibiotics $[1,2]$, thereby protecting bacterial cells against some non-optimal and lethal environmental conditions [3]. Incidences of SCVs have been reported in clinical specimens [4], livestock and even in foods [5].

Bacterial SCVs can be induced by cold stress [6], triclosan and living inside eukaryotic cells [1]. Additionally, exposure to antibiotics, like quinolones and aminoglycosides, has been shown to be able to induce bacterial SCVs $[7,8]$, resulting in an increased bacterial survival and ability to further proliferate. For example, streptomycin (STR), one of the aminoglycoside antibiotics, has been reported to induce SCVs of S. aureus [9]. STR, which was first used in plant agriculture in the 1950's, remains to be an important antibiotic for bacterial disease control in both animals and plants, especially in developing countries $[10,11]$. However, agricultural products, such as fruits, vegetables, livestock and poultry meats were found to be the most common sources of Salmonella enterica infections [12-14]. Therefore, Salmonella spp. SCVs may be induced by exposure to STR in agricultural products in these countries.

Salmonella spp. is a major causative agent of foodborne disease outbreaks, with Typhimurium as the most important serotype in outpatients with acute diarrhea [15]. Currently, SCV has been widely recognized to be part of the bacterial natural life cycle, including in Salmonella spp. [16]. Only a few literatures elucidated the genetic mechanisms for the development of S. Typhimurium SCVs by exposure to protamine or in the intracellular environment of fibroblasts. For example, mutations in the genes $l p d$, hemL, aroD, hemA and ubiA were reported to be responsible for the occurrence of $S$. Typhimurium SCVs $[16,17]$. The SCVs have a slow growth rate; however, these mutants are unstable that can revert to a phenotype of rapid growth at high frequency [18]. In S. enterica, only one genetic basis for SCV reversion was described by Maria et al. [19]. There can be multiple pathways involved in SCV reversion, and so far for a number of $S$. enterica revertants, the mechanism of reversion is still a speculation. Accordingly, it is of importance to disclose the full lifestyles of S. Typhimurium, its survival strategy, and to identify the genetic changes underlying the life cycle of $S$. Typhimurium SCVs.

In addition, some bacterial SCVs, such as Pseudomonas aeruginosa and S. aureus $[18,20]$, showed increased biofilm formation compared to their wild-type (WT) strains. The ability to form biofilms, which contributes to antibiotic resistance and protects bacteria against suboptimal environments, makes the pathogen extremely difficult to be eradicated $[21,22]$. Several studies have demonstrated biofilm formation of $S$. enterica in abiotic surfaces, plant surfaces and animal epithelial cells [23]. However, the ability of biofilm formation in the life cycle of SCVs in $S$. Typhimurium is still poorly understood.

In order to disclose the mode of S. Typhimurium survival after treated with STR and identify the genetic changes underlying the complete life cycle of $S$. Typhimurium SCVs, the fitness, antimicrobial susceptibility, biofilm formation, and the biofilm-related genes expression during the life cycle of SCVs were analyzed in comparison with their parent strains. Then whole genome sequencing of WT strain, $\mathrm{SCVs}$, and revertants in S. Typhimurium was performed to analyze the genetic changes in SCVs.

\section{Methods}

\section{Bacterial strains}

Selection of resistant mutants was performed as described previously [24] with some modifications. Briefly, WT strain 5, a susceptible clinical isolate obtained from stool sample from an infant with acute diarrhea in Shenzhen, China, was cultured at $37{ }^{\circ} \mathrm{C}$ on MuellerHinton agar (MHA) added with STR (Sigma-Aldrich, St. Louis, MO) to allow cells to undergo a multistep selection process to obtain SCV strains. STR was only present in the agar during the selection, starting at $16 \mu \mathrm{g} / \mathrm{mL}$ and increasing twofold each step to a maximum concentration of $1024 \mu \mathrm{g} / \mathrm{mL}$. A total of five S. Typhimurium SCVs (mutation rate: $5 \times 10^{-8}$ ) were obtained in vitro. Based on the results of biofilm formation analysis, two representative SCVs (1024-4 and 1024-5) were selected for further studies. In addition, one class of fast-growing strains (approximately $10 \%$ ) was observed on antibioticfree MHA agar after 5-day incubation at room temperature, and eight revertants reverted from strain 1024-4 or 1024-5 were collected. Two representative strains $4 \mathrm{~V} 1$ and 5V1, which reverted respectively from 1024-4 and 1024-5, were randomly selected for further studies. All the mutants were serially passaged on Salmonella chromogenic agar plates (CHROMagar, France) in the absence of STR for 7 days. The stock cultures were stored at $-80{ }^{\circ} \mathrm{C}$ in tryptone soy broth (TSB; Oxoid Ltd., Cambridge, UK) supplemented with $20 \%$ glycerol until use.

\section{Molecular subtyping of mutants}

The homology of all the mutants, including WT strain, SCVs and revertants, were identified by pulsed-field gel electrophoresis (PFGE; using XbaI) and multiple-locus variable-number tandem-repeat analysis (MLVA) following standard PulseNet protocols (http://www.cdc.gov/ pulsenet/pathogens/). Dendrograms of MLVA and PFGE 
patterns were then analyzed using Bionumerics software (Version No. 6.0; Applied Maths, Sint-Martens-Latem, Belgium).

\section{Transmission electron microscopy (TEM)}

Transmission electron microscopy was performed to assess the phenotypic characteristics at single cell level and the distribution of flagella, as described previously [25] with some modifications. In brief, 400-mesh carboncoated $\mathrm{Cu} / \mathrm{Rh}$ grids were incubated on $10 \mu \mathrm{L}$ of fresh culture grown overnight for $1 \mathrm{~min}$. After which, the excess media were removed by blotting off with filter paper. Each grid was dipped in $20-\mu \mathrm{L} 2 \%$ (w/v) phosphotungstic acid for $3 \mathrm{~min}$. Then the grids were air-dried. To count the flagella manually, grids were examined in a TEM (Hitachi HT7700) operated at $80.0 \mathrm{kV}$ and at a magnification of $10,000 \times$.

\section{Fitness}

In order to determine the growth of bacteria, WT strain, SCVs and revertants were incubated overnight in LuriaBertani broth (LB) at $37{ }^{\circ} \mathrm{C}$ and adjusted to obtain the same optical density at $595 \mathrm{~nm}$ by fresh LB. Then, bacteria were cultured in $\mathrm{LB}$ medium at $37^{\circ} \mathrm{C}$ for $15 \mathrm{~h}$ with shaking $(200 \mathrm{r} / \mathrm{min})$. The growth of bacteria in 96-well microplate was measured by Multiskan FC (Thermo Fisher Scientific Inc., USA) at $595 \mathrm{~nm}$ every $30 \mathrm{~min}$. Each isolate was conducted with eight replicates. The broth without inoculation was used as the blank control.

\section{Antimicrobial susceptibility tests}

Minimal inhibitory concentration (MIC) for 15 antimicrobial agents, including streptomycin (STR), imipenem (IMI), chloramphenicol (CHL), gentamicin (GEN), ciprofloxacin (CIP), trimethoprim/sulfamethoxazole (SXT), ceftriaxone (AXO), amoxicillin/clavulanic acid 2:1 ratio (AUG2), ampicillin (AMP), nalidixic acid (NAL), tetracycline (TET), cefoxitin (FOX), doxycycline (DOX), cefepime (FEP), and azithromycin (AZI), were determined by broth microdilution method using Thermo Scientific $^{\mathrm{TM}}$ Sensititre $^{\mathrm{TM}}$ susceptibility MIC plates (Thermo Scientific Inc., USA) following the manufacturer's instructions. Breakpoints for sensitive, intermediate and resistant were defined by the Clinical and Laboratory Standards Institute, 2012. The Escherichia coli ATCC 25922 was used as quality control.

\section{Biofilm formation assay}

Biofilm production was analyzed using crystal violet assay [26]. In passing, bacterial cultures grown at $37{ }^{\circ} \mathrm{C}$ with shaking $(200 \mathrm{r} / \mathrm{min})$ overnight were adjusted to the optical density $\left(\mathrm{OD}_{595}\right)$ of 0.01 by diluting with TSB
(1:10). Then, $100 \mu \mathrm{L}$ of the diluted bacterial suspension was added to each well and incubated at $28{ }^{\circ} \mathrm{C}$ for $48 \mathrm{~h}$ without shaking. The medium was then discarded, and each well was washed with distilled water and stained with $100 \mu \mathrm{L}$ of crystal violet for $15 \mathrm{~min}$. Finally, the wells were again washed for five times with distilled water, and the biofilm formation was assayed with addition of $100 \mu \mathrm{L}$ of $95 \%$ ethanol to determine the OD at $570 \mathrm{~nm}$ by Multiskan FC (Thermo Fisher Scientific Inc., USA). The TSB (1:10) was used as the negative control. Each isolate was conducted with eight replicates.

Confocal laser scanning microscopy (CLSM) was used to study biofilm structure in mutants with the ability to form biofilm as described by Bridier et al. [27] with some modifications. Overnight bacterial cultures were adjusted to an $\mathrm{OD}_{595}$ of 0.01 by diluting with TSB (1:10) before being suspended in a $35-\mathrm{mm}$ glass-bottom dish and incubated at $28{ }^{\circ} \mathrm{C}$ for $48 \mathrm{~h}$ without shaking. After the formation of biofilm, the dishes were washed five times with distilled water and refilled with diluted (1:10) TSB containing $5 \mu \mathrm{M}$ Syto9 (1: 1000 dilution from a Syto9 stock solution at $5 \mathrm{mM}$ in DMSO; Invitrogen, USA). The dishes were then incubated in the dark at $28{ }^{\circ} \mathrm{C}$ for $20 \mathrm{~min}$ to enable the fluorescent labeling of the bacteria. Images were acquired by CLSM (Leica microsystems Inc., Germany). Stack images were captured with a z-step of $1 \mu \mathrm{m}$ and then biofilm images were reconstructed into three-dimensional images (3D) by IMARIS 8.0 software. The number of pixels of green fluorescent in images was determined for substratum coverage of biofilm.

\section{Congo red phenotype}

The "rdar" (red, dry and rough) morphotype related to biofilm formation is indicative of curli fimbriae and cellulose production on Congo red plate. WT strain and all mutants were cultured overnight in TSB at $37^{\circ} \mathrm{C}$ before being normalized to the same OD at $595 \mathrm{~nm}$. Then, $10 \mu \mathrm{L}$ of each culture was spotted onto an LB plate (without $\mathrm{NaCl}$ ) containing $40 \mu \mathrm{g} / \mathrm{mL}$ Congo red, and further incubated for $48 \mathrm{~h}$ at $28^{\circ} \mathrm{C}$.

\section{RNA extraction and real-time PCR}

RNA were extracted from WT strain, SCVs and revertants bacterial cultures grown at $28{ }^{\circ} \mathrm{C}$ for $48 \mathrm{~h}$ using the RNeasy Mini Kit (Qiagen; Germany), which was later used to synthesize complementary DNA (PrimeScript ${ }^{\mathrm{TM}}$ cDNA Synthesis Kit; TaKara, China). The expression levels of biofilm-related genes $\operatorname{csg} B, \operatorname{csg} D$, adrA and bapA were determined by real-time PCR following standard protocols coupled with primers described by Fa'brega et al. [24]. Relative gene expression was evaluated using 
the $2^{-\triangle \triangle \mathrm{CT}}$ method, and the $16 \mathrm{~S}$ rRNA gene was used as reference gene. Each strain was tested in triplicate.

\section{Whole-genome resequencing and identification of genetic variations}

Genomic DNA of WT (strain 5), SCVs (strain 1024-4 and 1024-5) and revertants (4V1 and 5V1) was extracted from overnight cultures using a TIANamp bacteria DNA Kit (TIANGEN, China). Nucleic materials were sent to Beijing Genomics Institute (BGI) for whole genome sequencing using Illumina Hiseq $2000^{\mathrm{TM}}$ platform with an average of $100 \times$ coverage per bacterial genome. Quality-filtered reads for each strain were separately aligned to the assembled contigs of WT to determine variations using SOAPaligner ( $\mathrm{v} 2.21$ ) and BWA ( $\mathrm{v}$ 0.6.2-r126). Then the reads with indels and SNPs were mapped back to the reference genome of $S$. Typhimurium LT2 (RefSeq NC_003197.1). The indels and SNPs were further verified by PCR and Sanger sequencing method (Invitrogen, China). Also, the genetic alterations in the other revertants (strain $4 \mathrm{~V} 2,4 \mathrm{~V} 3,4 \mathrm{~V} 4,5 \mathrm{~V} 2,5 \mathrm{~V} 3$ and 5V4) were further identified by PCR and Sanger sequencing method.

\section{Statistical analysis}

All statistical analyses were performed using the software SPSS 13.0 for Windows as $P$ value $<0.05$ considered to be significant.

\section{Results}

Phenotypic characterization of S. Typhimurium SCVs and revertants

One clinical isolate of S. Typhimurium was selected as wild-type strain, and two SCV mutants induced by STR were successfully obtained in vitro. Moreover, two fastgrowing strains that reverted back from their corresponding SCVs were also obtained (Fig. 1a). WT strain and all the mutants showed identical PFGE and MLVA profiles (Additional file 1: Figure S1).

In order to study the phenotypic characteristics in the different life stages of S. Typhimurium SCV, the morphologies of colonies, the distribution of the number of flagella and the fitness were analyzed. The SCV colonies on Salmonella chromogenic agar plates appeared to be small compared with the wild-type parental strain and revertants (Representative data were shown in Fig. 1b). Based on TEM images, both strains $4 \mathrm{~V} 1$ and $5 \mathrm{~V} 1$ showed significantly more flagella compared to their SCV mutants (ANOVA, $P<0.05$; Additional file 1: Figure S2). Moreover, strain $4 \mathrm{~V} 1$ had significantly more flagella than the WT strain $(P<0.05)$. The SCVs of S. Typhimurium displayed slower growth rate in comparison with the WT strain, while the revertants and WT strain growing in LB obtained similar growth rate (Fig. 1c).

\section{Antimicrobial susceptibility test}

As resistance to a variety of antibiotics is one of the typical characteristics of SCVs, we determined the MICs of several antibiotics for WT strain, SCVs and revertants (Table 1). SCVs showed two- to fourfold increments of MICs for IMI and GEN, and a drastic 64-fold increase in STR when compared with the wild-type parental strain. The revertants also showed an increase of two- to fourfold in the MICs of STR and decrease of two- to fourfold in the MICs of AMP and AZI when compared with the wild-type parental strain.

\section{Biofilm formation}

Biofilm production was analyzed in WT strain, SCVs, and revertants (Fig. 2) and the biofilm structures of SCVs were presented in Fig. 2a. Substratum coverage of biofilm in SCVs (strain 1024-4 and 1024-5) was significantly bigger than WT strain $(P<0.01$; Fig. $2 b)$. Consistent with this, SCVs of strain 1024-4 and 1024-5 also exhibited significantly increased production of biofilm according to crystal violet assay $(P<0.01$; Fig. $2 \mathrm{c})$. However, this characteristic lost in all eight fast growing strains when SCVs reverted back to the normal phenotype $(P>0.05$; Additional file 1: Figure S3).

\section{Expression of biofilm-related genes in SCVs}

The relative expression of biofilm-related genes was analyzed in mutants with the ability to form biofilm by the RT-PCR. For strain 1024-4, expression of the gene $\operatorname{csg} B$ $(P<0.01)$ was 32 times higher (Fig. 3$)$, while a fourfold increase was observed in both $a d r A$ and bapA genes $(P<0.05)$, and no significant change was found in $c s g D$ in comparison with WT strain $(P>0.05)$. For strain 10245 , a significant increase was found in $\operatorname{csg} B$ (eightfold; $P<0.01$ ), but no significant change in $\operatorname{adr} A, \operatorname{csg} D$ and bapA $(P>0.05)$. Based on the Congo red staining, SCV strain (1024-4) showed rdar morphology which is indicative of curli and cellulose production.

\section{Genetic alterations}

To explore the genetic changes in the life cycle of SCVs, whole-genome sequences of the SCVs (strain 1024-4 and 1024-5) and revertants (strain $4 \mathrm{~V} 1$ and 5V1) were compared to the WT strain (strain 5). Only five mutations were identified in four mutants by analyzing the data of whole genome sequencing, and notably, three of them were associated with the gene $u b i E$ (Fig. $4 \mathrm{a}$ and Table 2). A four bp length deletion occurred in ubiE of SCV strain 1024-5, which might de-activate the gene through 
a

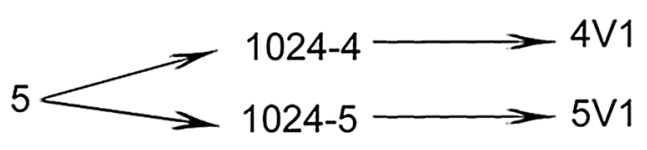

WT SCV Revertants

b
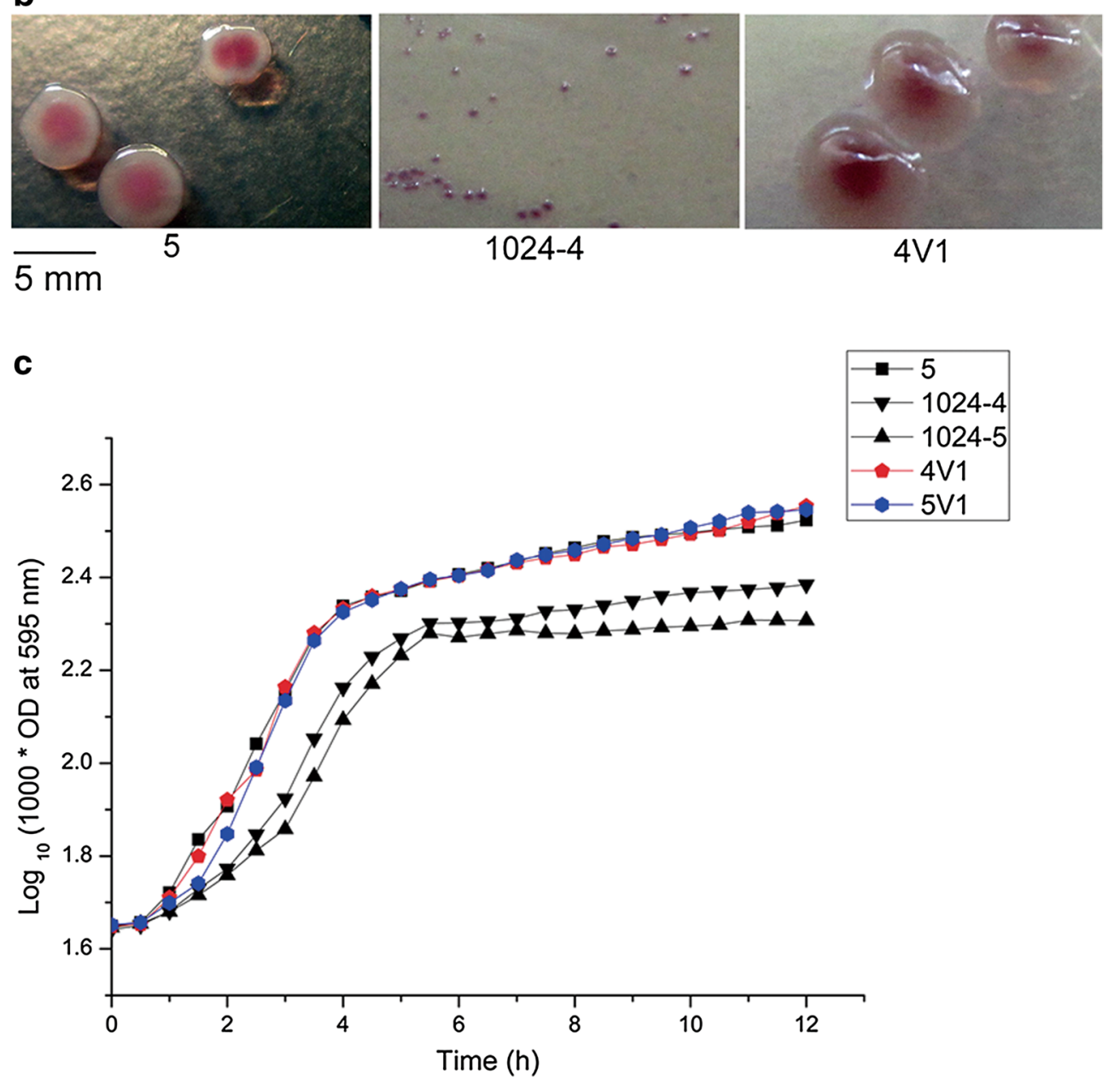

Fig. 1 Phenotypic characterization of WT, SCVs and revertants of S. Typhimurium. a The relationship between WT (wide-type; strain 5), SCVs (strain 1024-4 and 1024-5) and revertants (strain 4V1 and 5V1). b Representative data on the colony morphologies of strains on chromogenic agar plate. c Bacterial fitness evaluated by growth curves, and strains were incubated in Luria-Bertani broth (LB) at $37^{\circ} \mathrm{C}$ with shaking

Table 1 MIC determination in wide-type strain (WT), small colony variants (SCV), and revertants

\begin{tabular}{|c|c|c|c|c|c|c|c|c|c|c|c|c|c|c|c|}
\hline \multirow[t]{2}{*}{ Strain } & \multicolumn{15}{|c|}{$\mathrm{MIC}(\mu \mathrm{g} / \mathrm{mL})$} \\
\hline & STR & IMI & $\mathrm{CHL}$ & GEN & CIP & SXT & AXO & AUG2 & AMP & NAL & TET & FOX & DOX & FEP & AZI \\
\hline $5(\mathrm{WT})$ & 16 & $<0.5$ & $<2$ & $<1$ & $<0.03$ & $<0.12 / 2.38$ & $<0.25$ & $<1 / 0.5$ & 2 & 4 & $<2$ & 4 & 4 & $<1$ & 8 \\
\hline 1024-4 (SCV) & $>1024$ & 1 & $<2$ & 4 & $<0.03$ & $0.25 / 4.75$ & $<0.25$ & $2 / 1$ & 2 & 4 & $<2$ & 4 & 4 & $<1$ & $<2$ \\
\hline 1024-5 (SCV) & $>1024$ & 1 & $<2$ & 4 & $<0.03$ & $<0.12 / 2.38$ & $<0.25$ & $<1 / 0.5$ & $<1$ & 4 & $<2$ & 4 & 4 & $<1$ & $<2$ \\
\hline 4V1 (revertant) & 32 & $<0.5$ & 4 & $<1$ & $<0.03$ & $<0.12 / 2.38$ & $<0.25$ & $<1 / 0.5$ & $<1$ & 4 & $<2$ & 4 & 4 & $<1$ & 4 \\
\hline 5V1 (revertant) & 64 & $<0.5$ & 4 & 2 & $<0.03$ & $<0.12 / 2.38$ & $<0.25$ & $<1 / 0.5$ & $<1$ & 4 & $<2$ & 4 & 4 & $<1$ & 4 \\
\hline
\end{tabular}

STR streptomycin; IMI imipenem; CHL chloramphenicol; GEN gentamicin; CIP ciprofloxacin; SXT trimethoprim/sulfamethoxazole; AXO ceftriaxone; AUG2 amoxicillin/ clavulanic acid 2:1 ratio; $A M P$ ampicillin; NAL nalidixic acid; TET tetracycline; FOX cefoxitin; DOX doxycycline; FEP cefepime; $A Z I$ azithromycin 
a

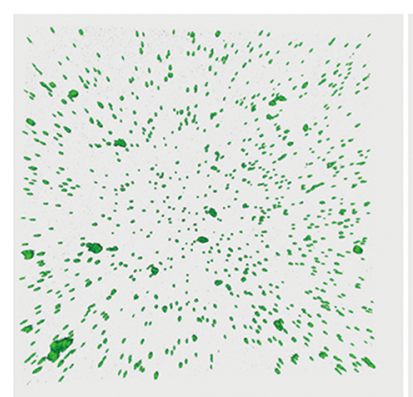

5

(WT)

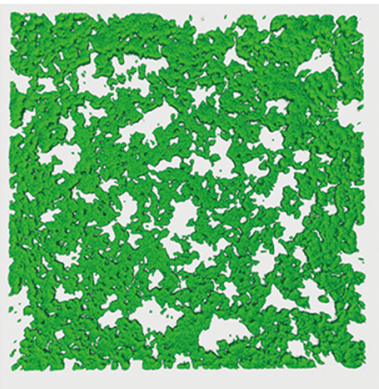

1024-4

(SCV)

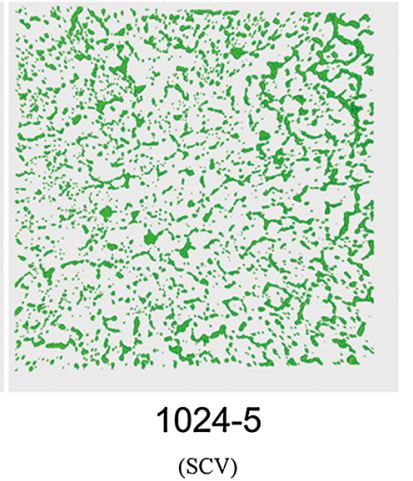

(SCV)
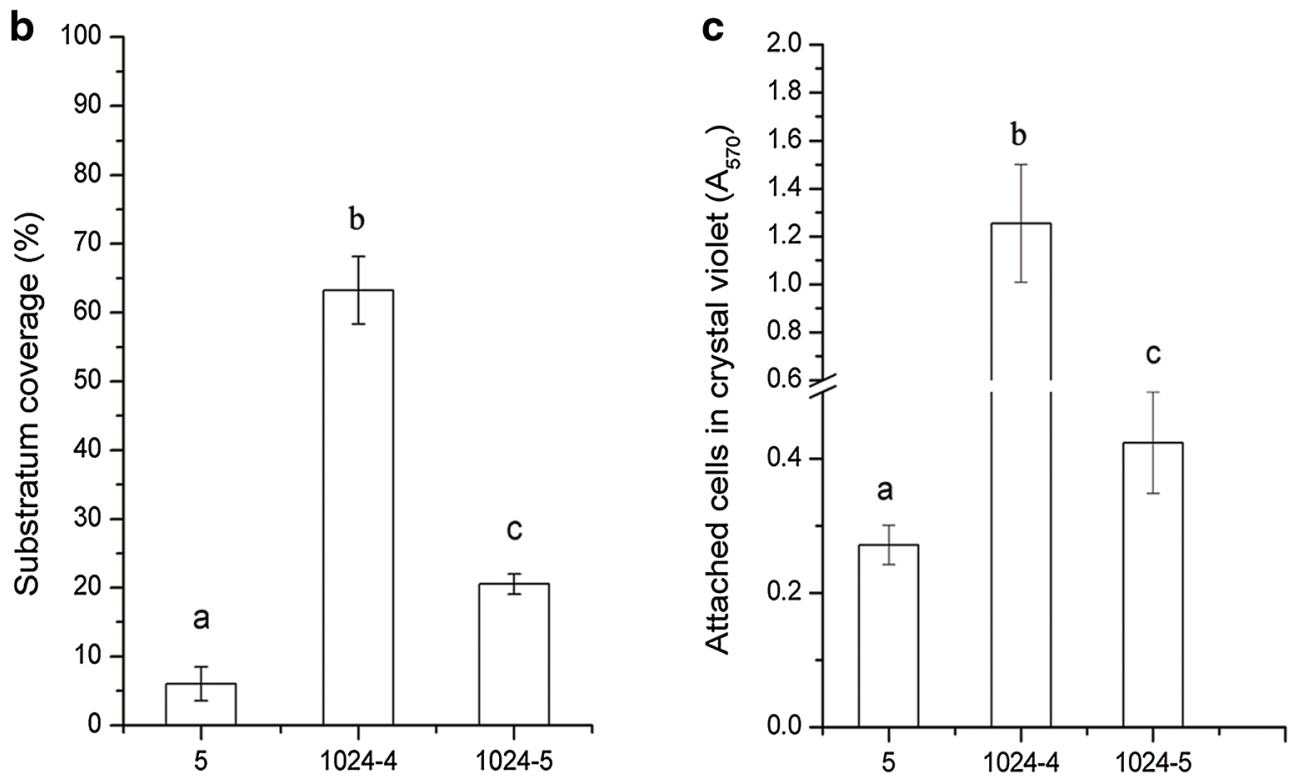

Fig. 2 Biofilm production of WT (wide-type; strain 5) and SCVs (strain 1024-4 and 1024-5). a A 3D projection of a biofilm structure of SCVs obtained by CLSM. b Ability of biofilm formation by using substratum coverage. c Ability of biofilm formation based on crystal violet assay. Data are presented as mean \pm SD. Different letters on the error bars indicate statistically significant differences between strains in each group based on ANOVA $(P<0.01)$

frameshift and premature termination (Fig. 4b). Interestingly, in its revertant strain, $5 \mathrm{~V} 1$, the second mutation in $u b i E$ (a one base pair insertion) recovered most of the amino acid sequence of the $u b i E$ and hence might re-activate this gene (Fig. 4b). A frameshift mutation in ubiE was also observed in another SCV strain, 1024-4, suggesting a close association between the activity of the $u b i E$ and the SCV phenotype (Table 2).

For strain 4V1, the revertant of 1024-4, carried two SNPs beside in addition to the defective ubiE (Fig. 4a). One SNP occurred in a putative gene that encodes for an inner membrane protein (Table 2). However, this SNP did not change the amino acid sequence of the product, and could then just be a neutral mutation. Another SNP in the gene $\operatorname{pr} f B$ led to a substitution of serine by leucine (S271L). This non-synonymous mutation could compensate the functional deficiency of mutated $u b i E$, resulting in the recovery of the phenotype (Fig. 4c).

Based on the results of PCR amplification and sequencing of mutated genes in the other revertants $(5 \mathrm{~V} 2,5 \mathrm{~V} 3$, $5 \mathrm{~V} 4,4 \mathrm{~V} 2,4 \mathrm{~V} 3$ and $4 \mathrm{~V} 4$ ), a total of two modes of genetic change for reversion to a rapidly growing form were observed. The revertants (strain 5V2, 5V 3 and 5V4) that reverted back from strain 1024-5, also harbored a secondary mutation in $u b i E$ (Fig. 4b), which reinstated most of the amino acid sequence of the $u b i E$. In addition, revertants (strain 4V2, 4V3, 4V4) from 1024-4 harbored a mutation in $\operatorname{prfB}$ (Fig. 4c). 


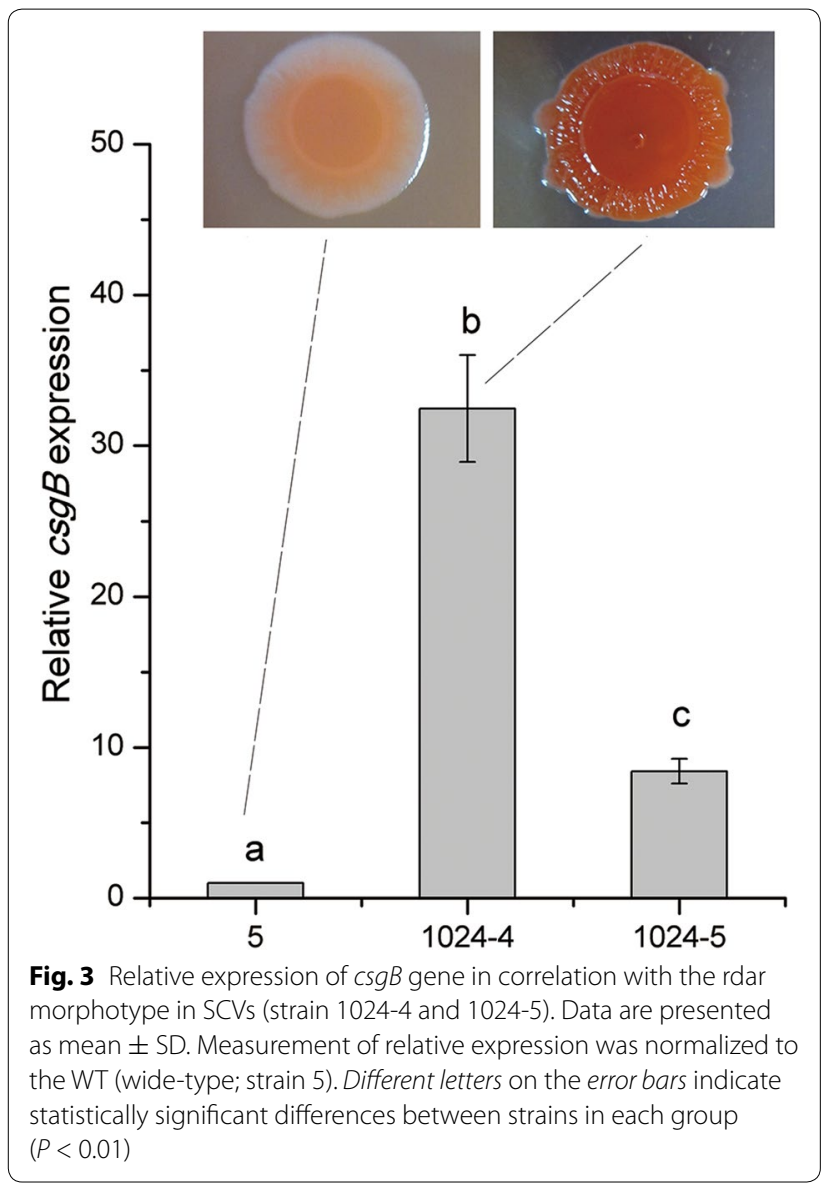

\section{Discussion}

Slower growth, reduced metabolic activity and increased antimicrobial resistance are typical characteristics for bacterial SCVs [28], which have been most extensively studied for S. aureus. In this study, S. Typhimurium SCVs obtained by the treatment of STR also displayed slower growth rate and increased antimicrobial resistance, additionally, with partially increased production of biofilm in comparison to the WT strain.

From the perspectives of food industry, the occurrence of S. Typhimurium SCV may increase the potential infection risks. Since STR is still widely used in farm industry in developing countries, exposure to STR could result in the occurrence of S. Typhimurium SCVs. Slower growth and reduced metabolic activity are typical characteristics for SCVs of many bacteria. Conventional identification tests (i.e. biochemical tests) are hampered or modified by the decreased metabolism of these SCVs giving no reaction or false negative results $[1,29]$, which may complicate the routine isolation and identification of bacteria for laboratory-based surveillance of contamination in food. In this way, the risk of Salmonella infection might become increased.
Clinically, the phenotypes of SCVs are associated with persistent and recurrent infections in $S$. aureus and $P$. aeruginosa due to their increased ability to persist in mammalian cells and increased antimicrobial resistance [2]. In S. aureus, Long et al. have demonstrated that the minimum bacteriocidal concentration was two- to fourfold higher than the parental strain, which may correlate to the bacterial persister cells, however, there was very little change in MIC for SCVs [30]. Consistently, a slightly increase in MIC of IMI and GEN was observed in S. Typhimurium SCV mutants. In addition, these SCVs remained susceptible to critical antimicrobials, such as AUG2, AMP and CIP. These data confirm that switching to the SCVs phenotype does not bring a great change in MIC. Additionally, there was a great increase in STR resistance as these SCVs were selected by STR.

In most bacteria, the ability to form biofilm contributes to conferring tolerance and firm adhesion, which helps the bacteria to survive in suboptimal environments, making it extremely difficult to be eradicated. Therefore, the food borne pathogenic bacteria with the ability to form biofilm may threaten the safety of food industry [31]. In this study, a total of five S. Typhimurium SCVs were obtained, among which two mutants (strain 1024-4 and 1024-5) displayed a significantly increased biofilm formation in comparison to their wild type strain (ANOVA, $P<0.05$; data not shown). Consistent with this, approximately $30 \%$ of $P$. aeruginosa SCVs was observed to exhibit increased attachment to glass by Drenkard et al. [32]. Several genes, including $\operatorname{csg} D, a d r A, \operatorname{csg} B$ and $b a p A$, have been identified to be associated with biofilm formation in S. enterica and they were quantified by RT-PCR in this study. $\operatorname{csg} D$, a transcriptional regulator, decides whether S. enterica strains would form biofilms [33]. On the other hand, the gene $a d r A$, which controls the levels of cyclic di-GMP, is related to cellulose production, and $\operatorname{csg} B$ is the nucleator of the curli fimbriae [34]. In addition, BapA is a large secreted protein required for biofilm formation in $S$. Enteritidis [35]. In our study, $c \operatorname{sg} B$ expressed at a much higher level in SCVs, compared with the WT strain. Particularly, the relative expression of $\operatorname{csg} B$ reached a maximum of 32-fold in strain 1024-4, suggesting that "rdar" morphotype and biofilm production observed in this strain were possibly attributable to an increased expression of this gene. The associated production of cellulose and curli fimbriae have been well characterized as the "rdar" morphotype which is related to biofilm formation in S. enterica [36].

Coenzyme $\mathrm{Q}$ is a redox lipid that functions in the respiratory electron transport chain, and also plays a critical role in energy generating processes [37]. Biosynthesis of coenzyme Q in $E$. coli depends on the $u b i$ genes [38]. The $u b i E$ gene encodes ubiquinone/menaquinone 


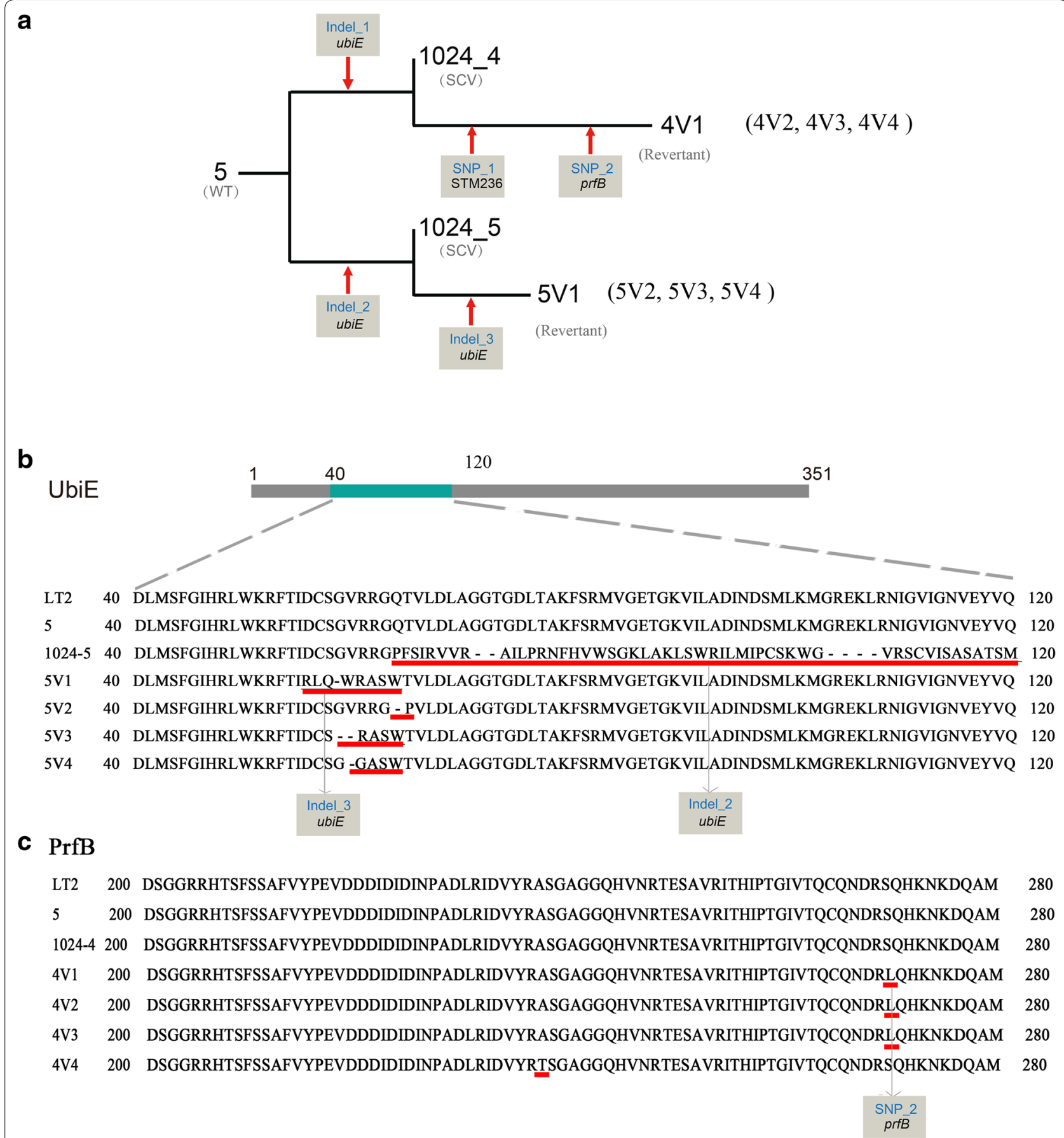

Fig. 4 Two modes of genetic change for reversion to a rapidly growing form in SCVs. a Whole-genome resequencing was performed in wide-type strain (WT, strain 5), SCVs (strain 1024-4 and 1024-5) and revertants (strain 4V1 and 5V1) and the genetic alterations in the other revertants (strain $4 \mathrm{~V} 2,4 \mathrm{~V} 3,4 \mathrm{~V} 4,5 \mathrm{~V} 2,5 \mathrm{~V} 3$ and $5 \mathrm{~V} 4$ ) were further identified by PCR and Sanger sequencing method. $\mathbf{b}$ Amino acid sequence alignment of UbiE for reference strain (S. Typhimurium LT2), WT, SCVs and revertants. c Amino acid sequence alignment of PrfB for reference strain (S. Typhimurium LT2), WT, SCVs and revertants

biosynthesis methyltransferase, which is required for C-methylation reactions in both coenzyme $\mathrm{Q}_{8}$ and menaquinone biosynthesis [39]. In this study, a frameshift mutation in $u b i E$ was observed in all SCVs, which possibly led to the deficiency of electron-transport and the formation of SCV phenotype. As previously reported, 
Table 2 List of genetic changes in S. Typhimurium SCVs and revertants

\begin{tabular}{|c|c|c|c|c|c|c|}
\hline Mutation $^{a}$ & LT2 Position ${ }^{\mathbf{b}}$ & Mutant strain & Mutant type & Amino acid change & Gene name & Gene product \\
\hline Indel_1 & $4,177,676$ & $1024 \_4,4 \mathrm{~V} 1$ & Insertion: $T$ & Frame shift & ubiE (STM3970) & $\begin{array}{l}\text { Ubiquinone/menaquinone Biosynthesis } \\
\text { C-methyltransferase UbiE }\end{array}$ \\
\hline Indel_2 & $4,177,484: 4,177,487$ & 1024_5,5V1 & Deletion: GTTT & Frame shift & ubiE (STM3970) & $\begin{array}{l}\text { Ubiquinone/menaquinone biosynthesis } \\
\text { C-methyltransferase UbiE }\end{array}$ \\
\hline Indel_3 & $4,177,459$ & $5 \mathrm{~V} 1$ & Insertion: $T$ & Frame shift & ubiE (STM3970) & $\begin{array}{l}\text { Ubiquinone/menaquinone biosynthesis } \\
\text { C-methyltransferase UbiE }\end{array}$ \\
\hline SNP_1 & $2,480,126$ & $4 \mathrm{~V} 1$ & SNP: G to $A$ & F125F & STM2367 & Inner membrane protein \\
\hline SNP_2 & $3,201,008$ & $4 \mathrm{~V} 1$ & SNP: G to $A$ & S271L & prfB (STM3041c) & Peptide chain release factor 2 \\
\hline
\end{tabular}

a The genetic changes in SCVs and revertants were compared to the wild-type parental strain by analyzing the data of whole genome sequencing

b Corresponding position in the reference genome of S. Typhimurium, LT2 (Accession number: NC_003197.1)

the biogenesis of flagella would be inhibited in the strains bearing deletion mutation of $u b i E$ due to the lack of energy source [40]. However, complete deletion of $u b i E$ is not naturally occurring in any life stages of bacteria. In this study, SCVs displayed less flagella with a mutation that naturally occurred in $u b i E$, which may be due to the reduced energy generating for flagellar biogenesis [41].

Fitness costs can be offset by compensatory mutations. In this study, two modes of the genetic changes for reversion to the rapidly growing form were observed. All the four revertants that reverted back from strain 1024-5, harbored secondary mutations in $u b i E$. It was possible that the secondary changes in the protein-coding domain led to recovery on the electron-transport compared with SCV strain (strain 1024-5). Moreover, another mode of genetic change for reversion to a rapidly growing form was observed in the revertants reverting back from 1024-4 (strain 4V1, 4V2, 4V3, 4V4). All the revertants harbored a mutation in $\operatorname{prf} B$ (encoding for peptide chain release factor 2), suggesting that the deficiency in electron-transport could be compensated by the mutation of $\operatorname{prfB}$ gene. Peptide chain release factor 2 gene, $\operatorname{prf} B$, was catalyzes translational termination at UGA or UAA codons [42], but how the mutation in $\operatorname{prf} B$ compensated the growth cost of SCVs needs to be further investigated.

\section{Conclusion}

To conclude, this study disclosed the specific genetic changes that involved in the life cycle of SCVs in S. Typhimurium, which helps to get a better understanding of the genetic basis for the ability of SCVs to revert to the phenotype of rapid growth. S. Typhimurium could switch to the phenotype of SCV by a mutation in $u b i E$ under the treatment of STR. Moreover, the available biofilm formation of S. Typhimurium SCVs may be a strategy for survival. Therefore, the rational use of STR in agriculture or farm industry should be considered to reduce the potential risk of S. Typhimurium infection. Under a favorable environmental condition, these SCVs can escape from the growth restriction by a second-site compensatory mutation in $\operatorname{prfB}$ or a new mutation in $u b i E$. These genetic changes (SNPs and indels) that occurred in the life cycle of S. Typhimurium SCVs were different to those previously reported in the literature. And these findings may contribute to developing phenotypedirected treatments against $S$. Typhimurium SCVs.

\section{Additional file}

Additional file 1: Figure S1. Dendrograms of wide-type, SCVs and revertants evaluated by MLVA and PFGE. Figure S2. Representative data on transmission electron microscopy analysis of strain morphology and the number of flagella. Figure S3. Ability of biofilm formation of revertants and WT strain.

\begin{abstract}
Abbreviations
S. Typhimurium: Salmonella enterica serotype Typhimurium; SCVs: small colony variants; STR: streptomycin; WT: wild-type strains; MHA: Mueller-Hinton agar; TSB: tryptone soy broth; PFGE: pulse field gel electrophoresis; MLVA: multilocus variable number tandem repeat analysis; CDC: center for disease control and prevention; TEM: transmission electron microscopy; PCR: polymerase chain reaction; SNP: single-nucleotide polymorphism; LB: Luria-Bertani broth; MIC: minimal inhibitory concentration; IMI: imipenem; CHL: chloramphenicol; GEN: gentamicin; CIP: ciprofloxacin; SXT: trimethoprim/sulfamethoxazole; AXO: ceftriaxone; AUG2: amoxicillin/clavulanic acid 2:1 ratio; AMP: ampicillin; NAL: nalidixic acid; TET: tetracycline; FOX: cefoxitin; DOX: doxycycline; FEP: cefepime; AZI: azithromycin; CLSM: confocal laser scanning microscopy; cDNA: complementary DNA; rdar: red, dry and rough.
\end{abstract}

\section{Authors' contributions}

All authors contributed to this work. All authors read and approved the final manuscript.

\section{Author details}

${ }^{1}$ Key Laboratory of Bio-resources and Eco-environment of the Ministry of Education, College of Life Sciences, Sichuan University, 29\# Wangjiang Rd., Chengdu, Sichuan 610064, P. R. China. ${ }^{2}$ Shenzhen Major Infectious Disease Control Key Laboratory, Shenzhen Centre for Disease Control and Prevention, Shenzhen, Guangdong 518055, P. R. China. ${ }^{3}$ State Key Laboratory of Pathogen and Biosecurity, Beijing Institute of Microbiology and Epidemiology, Beijing 100071, P. R. China. 


\section{Acknowledgements}

This research was supported by the China National Science and Technology Major Projects Foundation (2012ZX10004215-003-005), the Department of Science and Technology of Sichuan Province of China (2016NZ005, 2016SZ0068).

\section{Competing interests}

The authors declare that they have no competing interests.

Received: 1 April 2016 Accepted: 18 May 2016

Published online: 31 May 2016

\section{References}

1. Kahl BC. Small colony variants (SCVs) of Staphylococcus aureus-a bacterial survival strategy. Infect Genet Evol. 2014;21:515-22.

2. Proctor RA, von Eiff C, Kahl BC, Becker K, McNamara P, Herrmann M, Peters G. Small colony variants: a pathogenic form of bacteria that facilitates persistent and recurrent infections. Nat Rev Microbiol. 2006;4(4):295-305.

3. Yagci S, Hascelik G, Dogru D, Ozcelik U, Sener B. Prevalence and genetic diversity of Staphylococcus aureus small-colony variants in cystic fibrosis patients. Clin Microbiol Infec. 2013;19(1):77-84.

4. Anderson SW, Stapp JR, Burns JL, Xuan Q. Characterization of smallcolony-variant Stenotrophomonas maltophilia isolated from the sputum specimens of five patients with cystic fibrosis. J Clin Microbiol. 2007;45(2):529-35.

5. Atalla H. Characterization of a Staphylococcus aureus small colony variant (SCV) associated with persistent bovine mastitis. Foodborne Pathog Dis. 2008;5(6):785-99.

6. Onyango LA, Dunstan RH, Gottfries J, Von EC, Roberts Tk. Effect of low temperature on growth and ultra-structure of Staphylococcus spp. PLoS One. 2012;7(1):339.

7. Xiao-Su P, Hamlyn PJ, Raquel TV, Alovero FL, Manzo RH, L Mark F. Smallcolony mutants of Staphylococcus aureus allow selection of gyrase-mediated resistance to dual-target fluoroquinolones. Antimicrob Agents Ch. 2002;46(8):2498-506.

8. Schaaff F, Bierbaum G, Baumert N, Bartmann P, Sahl HG. Mutations are involved in emergence of aminoglycoside-induced small colony variants of Staphylococcus aureus. Int J Med Microbiol. 2004;293(6):427-35.

9. Massey RC, Buckling A, Peacock SJ. Phenotypic switching of antibiotic resistance circumvents permanent costs in Staphylococcus aureus. Curr Biol. 2001;11(22):1810-4.

10. Wen-Hong LI, Cheng Y, Jin JX, Feng-Liang LI. History and research advance of streptomycin for controlling plant bacterial diseases. Hubei Agricultural Sciences 2013. (In Chinese).

11. Door APDL, Chacón AR, Muñiz CA. Detection of streptomycin resistance in Erwinia amylovora strains isolated from apple orchards in Chihuahua, Mexico. Eur J Plant Pathol. 2013;137(2):223-9.

12. Patel J, Sharma M. Differences in attachment of Salmonella enterica serovars to cabbage and lettuce leaves. Int J Food Microbiol. 2010;139(1-2):41-7.

13. Bolton DJ, Ivory C, McDowell D. A study of Salmonella in pigs from birth to carcass: serotypes, genotypes, antibiotic resistance and virulence profiles. Int J Food Microbiol. 2013;160(3):298-303.

14. Singh R, Yadav AS, Tripathi V, Singh RP. Antimicrobial resistance profile of Salmonella present in poultry and poultry environment in north India. Food Control. 2013;33(2):545-8.

15. Liang Z, Ke B, Deng X, Liang J, Ran L, Lu L, He D, Huang Q, Ke C, Li Z, et al. Serotypes, seasonal trends, and antibiotic resistance of non-typhoidal Salmonella from human patients in Guangdong Province, China, 2009-2012. BMC Infect Dis. 2015;15:53.

16. Cano DA, Pucciarelli MG, Martinez-Moya M, Casadesus J, Garcia-del Portillo F. Selection of small-colony variants of Salmonella enterica serovar typhimurium in nonphagocytic eucaryotic cells. Infect Immun. 2003;71(7):3690-8.

17. Koskiniemi S, Pränting M, Gullberg E, Näsvall J, Andersson DI. Activation of cryptic aminoglycoside resistance in Salmonella enterica. Mol Microbiol. 2011:80(6):1464-78
18. Eliana N, Ausubel FM. Pseudomonas biofilm formation and antibiotic resistance are linked to phenotypic variation. Nature. 2002;416(6882):740-3.

19. Pränting $M$, Andersson DI. Escape from growth restriction in small colony variants of Salmonella typhimurium by gene amplification and mutation. Mol Microbiol. 2011;79(2):305-15

20. Singh R, Ray P, Das A, Sharma M. Enhanced production of exopolysaccharide matrix and biofilm by a menadione-auxotrophic Staphylococcus aureus small-colony variant. J Med Microbiol. 2010;59(5):521-7.

21. Nguyen HDN, Yuk H-G. Changes in resistance of Salmonella typhimurium biofilms formed under various conditions to industrial sanitizers. Food Control. 2013;29(1):236-40.

22. Vázquez-Sánchez D, Cabo ML, Ibusquiza PS, Rodríguez-Herrera JJ. Biofilmforming ability and resistance to industrial disinfectants of Staphylococcus aureus isolated from fishery products. Food Control. 2014;39:8-16.

23. Steenackers H, Hermans K, Vanderleyden J, De Keersmaecker SCJ. Salmonella biofilms: an overview on occurrence, structure, regulation and eradication. Food Res Int. 2012:45(2):502-31.

24. Fabrega A, Soto SM, Balleste-Delpierre C, Fernandez-Orth D, Jimenez de Anta MT, Vila J. Impact of quinolone-resistance acquisition on biofilm production and fitness in Salmonella enterica. J Antimicrob Chemoth. 2014;69(7):1815-24

25. van Ditmarsch D, Boyle Kerry E, Sakhtah H, Oyler Jennifer E, Nadell Carey D, Déziel É, Dietrich Lars EP, Xavier Joao B. Convergent evolution of hyperswarming leads to impaired biofilm formation in pathogenic bacteria. Cell Rep. 2013;4(4):697-708.

26. Ben-Jacob E, Bogino P, Abod An, Nievas F, Giordano W. Water-limiting Conditions alter the structure and biofilm-forming ability of bacterial multispecies communities in the alfalfa rhizosphere. PLoS One. 2013;8(11):e79614.

27. Bridier A, Dubois-Brissonnet F, Boubetra A, Thomas V, Briandet R. The biofilm architecture of sixty opportunistic pathogens deciphered using a high throughput CLSM method. J Microbiol Meth. 2010;82(1):64-70.

28. Eiff CV, Peters $G$, Becker K. The small colony variant (SCV) concept-the role of staphylococcal SCVs in persistent infections. Injury. 2006;37(2):S26-33.

29. Von EC, Mcnamara PK, Bates D, Lei XH, Ziman M, Bochner BR, Peters G, Proctor RA. Phenotype microarray profiling of Staphylococcus aureus menD and hemB mutants with the small-colony-variant phenotype. J Bacteriol. 2006;188(2):687-93.

30. Bui LMG, Kidd SP. A full genomic characterization of the development of a stable small colony variant cell-type by a clinical Staphylococcus aureus strain. Infect Genet Evol. 2015;36:345-55.

31. Srey S, Jahid IK, Ha SD. Biofilm formation in food industries: a food safety concern. Food Control. 2013;31(31):572-85.

32. Drenkard E, Ausubel FM. Pseudomonas biofilm formation and antibiotic resistance are linked to phenotypic variation. Nature. 2002;416(6882):740-3.

33. Chambers JR, Sauer K. Small RNAs and their role in biofilm formation. Trends Microbiol. 2013;21(1):39-49.

34. Grantcharova N, Peters V, Monteiro C, Zakikhany K, Romling U. Bistable expression of CsgD in biofilm development of Salmonella enterica serovar typhimurium. J Bacteriol. 2009;192(2):456-66.

35. Latasa C, Roux A, Toledo-Arana A, Ghigo JM, Gamazo C, Penadés JR, Lasa I. BapA, a large secreted protein required for biofilm formation and host colonization of Salmonella enterica serovar Enteritidis. Mol Microbiol. 2005:58(5):1322-39.

36. Römling U, Bokranz W, Rabsch W, Zogaj X, Nimtz M, Tschäpe H. Occurrence and regulation of the multicellular morphotype in Salmonella serovars important in human disease. Int J Med Microbiol. 2003;293(4):273-85

37. Laurent $A$, Laurent $L$, Mahmoud HC, Bérengère $P$, Marc F, Fabien $P$, Frédéric B. ubiJ, a new gene required for aerobic growth and proliferation in macrophage, is involved in coenzyme Q biosynthesis in Escherichia coli and Salmonella enterica Serovar typhimurium. J Bacteriol. 2014;196(1):70-9.

38. Gulmezian M, Hyman KR, Marbois BN, Clarke CF, Javor GT. The role of UbiX in Escherichia coli coenzyme Q biosynthesis. Arch Biochem Bioph. 2007:467(2):144-53. 
39. Aussel L, Loiseau L, Hajj Chehade M, Pocachard B, Fontecave M, Pierre F, Barras F. ubiJ, a new gene required for aerobic growth and proliferation in macrophage, is involved in coenzyme $\mathrm{Q}$ biosynthesis in Escherichia coli and Salmonella enterica serovar typhimurium. J Bacteriol. 2013;196(1):70-9.

40. Barker CS, Meshcheryakova IV, Sasaki T, Roy MC, Sinha PK, Yagi T, Samatey FA: Randomly selected suppressor mutations in genes for NADH

: quinone oxidoreductase-1, which rescue motility of a Salmonella ubiquinone-biosynthesis mutant strain. Microbiology. 2014; 160(Pt_6):1075-86.
41. Chevance FF, Hughes KT. Coordinating assembly of a bacterial macromolecular machine. Nat Rev Microbiol. 2008;6(6):455-65.

42. Rengby O, Arner ES. Titration and conditional knockdown of the prfB gene in Escherichia coli: effects on growth and overproduction of the recombinant mammalian selenoprotein thioredoxin reductase. Appl Environ Microbiol 2007;73(2):432-41.

\section{Submit your next manuscript to BioMed Central and we will help you at every step:}

- We accept pre-submission inquiries

- Our selector tool helps you to find the most relevant journal

- We provide round the clock customer support

- Convenient online submission

- Thorough peer review

- Inclusion in PubMed and all major indexing services

- Maximum visibility for your research

Submit your manuscript at

www.biomedcentral.com/submit 